\title{
Lyme borreliosis: a review of data on transmission time after tick attachment
}

This article was published in the following Dove Press journal:

International Journal of General Medicine

19 December 2014

Number of times this article has been viewed

\author{
Michael J Cook \\ Independent researcher, Dorset, UK
}

Correspondence: Michael ] Cook

39 Merley Drive, Highcliffe,

Dorset $\mathrm{BH} 23$ 5BN, UK

Tel +44 I425270832

Email mcook98@msn.com

\begin{abstract}
Lyme borreliosis is increasing rapidly in many parts of the world and is the most commonly occurring vector-borne disease in Europe and the USA. The disease is transmitted by ticks of the genus Ixodes. They require a blood meal at each stage of their life cycle and feed on a wide variety of wild and domestic animals as well as birds and reptiles. Transmission to humans is incidental and can occur during visits to a vector habitat, when host mammals and their associated ticks migrate into the urban environment, or when companion animals bring ticks into areas of human habitation. It is frequently stated that the risk of infection is very low if the tick is removed within 24-48 hours, with some claims that there is no risk if an attached tick is removed within 24 hours or 48 hours. A literature review has determined that in animal models, transmission can occur in $<16$ hours, and the minimum attachment time for transmission of infection has never been established. Mechanisms for early transmission of spirochetes have been proposed based on their presence in different organs of the tick. Studies have found systemic infection and the presence of spirochetes in the tick salivary glands prior to feeding, which could result in cases of rapid transmission. Also, there is evidence that spirochete transmission times and virulence depend upon the tick and Borrelia species. These factors support anecdotal evidence that Borrelia infection can occur in humans within a short time after tick attachment.
\end{abstract}

Keywords: Borrelia burgdorferi sensu lato, disease transmission, tick-borne disease, Lyme disease, tick attachment

\section{The disease}

Lyme borreliosis (LB) is a multisystemic disease that can affect many organs, including the heart, joints, central nervous system, and brain, and can result in a large number of diverse symptoms including extreme fatigue, flu-like symptoms, arthritis, peripheral neuropathy, and cognitive dysfunction. The disease is named after the town of Lyme in Connecticut, USA, where a group of children with an unusual arthritic condition were studied. The causal agent was identified by Willie Burgdorfer as a spirochetal bacterium, and named after him as Borrelia burgdorferi. ${ }^{1}$

The disease was first described over 100 years ago in Europe from the characteristic skin rash erythema chronicum migrans in the early stage and from the skin rash acrodermatitis chronica atrophicans that can occur in late stage of LB and named by Afzelius and Buchwald, respectively. ${ }^{2,3}$ The disease causing spirochetes are carried from reservoir host mammals to humans by hard-bodied ticks of the genus Ixodes. More than 18 species of Borrelia are known that are transmitted by Ixodes ticks, with the recently identified Borrelia kurtenbachii named after Klaus 
Kurtenbach. ${ }^{4}$ Three have been considered important human pathogens, Borrelia burgdorferi sensu stricto in the USA and Europe, and Borrelia afzelii and Borrelia garinii in Europe and Asia. ${ }^{5}$ Potential pathogenic Borrelia species include Borrelia bavariensis, Borrelia bissettii, B. kurtenbachii, Borrelia Spielmanii, Borrelia lusitaniae, and Borrelia valaisiana. ${ }^{6}$ The pathogenic Borrelia miyamotoi was identified in Japan, ${ }^{7}$ and is now known to be present in the USA and Russia, ${ }^{8}$ and recently discovered in the UK. ${ }^{9}$ Though genetically more closely related to relapsing fever (RF) disease, B. miyamotoi unlike RF is carried by hard-bodied Ixodes species ticks and in humans can result in LD like symptoms. The proportion of ticks carrying LB can vary widely and in one study of eleven regions of Switzerland between $9 \%$ and $47 \%$ of ticks were found to be infected. ${ }^{10}$ In a 2011 paper by Brown (unpublished data) presented at the 10th Tick Borne Disease conference hosted by Lyme Disease Action in the UK, tick populations were monitored over a number of years showing dramatic growth in population density with a threefold increase in feeding females from 1994 to 2004, and a 20-fold increase in Borrelia infected ticks from 1991 to 2009.

Although LB receives considerable attention and is the focus of this study, ticks transmit many diseases with 12 viral infections discussed by Lani et al including tick-borne encephalitis, Louping-ill, Colorado tick fever, and Alkhurma hemorrhagic fever, which has a mortality rate of $25 \% .{ }^{11}$ Hard-bodied ticks of the genus Ixodes also carry bacterial and parasitic diseases including: anaplasmosis, babesiosis, ehrlichiosis, rickettsiosis, and bartonellosis. Zhang et al investigated the microbiome of Ixodes persulcatus using rRNA sequencing and found 237 bacteria genera suspected of being pathogens to vertebrates. ${ }^{12}$ Many studies have found ticks infected with two or more of these pathogens and this increase in pathogen burden can result in more serious symptoms and post-treatment sequelae. ${ }^{13-16}$ An important emerging pathogen is Candidatus neoehrlichia mikurensis, ${ }^{17}$ and in a survey of Canadian residents, $62 \%$ of respondents reported at least one coinfection and $15 \%$ reported three coinfections with Bartonella and Babesia the most common. ${ }^{18}$ The attachment time for transmission of almost all of these pathogens is unknown; however, there are studies that indicate rapid transmission of some. Ebel and Kramer demonstrated Powassan virus infection with 15 minutes of tick attachment ${ }^{19}$ and although Saraiva et al found that transmission of Rickettsia rickettsii by unfed Amblyomma aureolatum ticks required $>10$ hours attachment time, they found that transmission could occur in as little as 10 minutes with fed ticks. ${ }^{20}$

\section{Incidence}

In Europe, few countries have made LB a notifiable disease, and lack of standards and changes in reporting and testing methodologies have made it difficult to obtain reliable incidence data. ${ }^{21} \mathrm{~A}$ World Health Organization report published in 2006 indicated that approximately 85,000 cases of LB were reported annually for Europe and that it was the most common zoonosis in temperate regions of the Northern Hemisphere. ${ }^{21}$ Estimates for Germany indicate that in 2007 and 2008, the average annual incidence of LB was 261 cases per 100,000 population..$^{22}$ This was based on the medical insurance records of 6.04 million patients in six north-eastern federal states, and by extrapolation generates an estimate of 213,912 cases annually for the country as a whole at that time. In the US, the Centers for Disease Control and Prevention reported an incidence of nearly 30,000 cases for 2009 based on specific case definitions for disease surveillance. Total LB infections were considered to be much higher, and a recent Centers for Disease Control and Prevention report gives an estimate of 300,000 cases based on three separate studies that included analysis of insurance claims and surveys of clinical laboratories and the general public, and states that the disease "is a tremendous public health problem $[\ldots]{ }^{2} .{ }^{23}$

\section{Attachment time and transmission risk}

It is commonly stated in health agency guidelines and the general media that the risk of LB increases with length of time a tick is attached. Additionally there are many claims that it requires attachment times $>24$ hours for transmission of infection to occur, and sometimes it requires 48 hours of attachment. Usually, these statements are made without reference to any data source, and so a comprehensive search and review of articles has been carried out to determine the original sources for these claims.

An early source is an article by Falco et al where it is stated that "... removal of attached Ixodes scapularis ticks within 48 hours will effectively prevent Lyme disease". ${ }^{24}$ The authors cited work with animal models to support the statement including a 1987 paper by Piesman et al. ${ }^{25}$ In a 2001 article, it was stated that I. scapularis ticks infected with B. burgdorferi sensu stricto removed during the first 2 days of attachment do not transmit infection to tick bite victims. ${ }^{26}$ 
A review of this work and extensive search for sources that confirmed this data was carried out.

\section{Tick feeding mechanisms}

Ixodes ticks have three feeding stages in their life cycle and take a blood meal at each. As larvae, nymphs, and adults, they seek a host by climbing the stems of grasses, for example, and when actively seeking a host, adopt a "questing" position, where the front legs extend ready to grasp a passing animal. After transferring to the host, the tick searches for a suitable attachment site. With natural hosts, this is typically in the head area giving access to the ears and neck region or groin area. ${ }^{27}$ Tick attachment starts by inserting the saw-like chelicerae into the epidermis to create an entrance wound. Then using the leverage from the chelicerae the barbed hypostome mouthpart is pulled in. This process is continued in a ratchet-like fashion until the tick is fully attached, with the process complete within 10 minutes. This is described by Richter et al, ${ }^{28}$ and demonstrated in a video. ${ }^{29}$ The multiple barbs of the hypostome lock the tick into the epidermis and help prevent host activities, for example, grooming, from detaching the tick, and in some species, a cement plug is created to further ensure firm attachment.

During attachment, ticks inject a complex mixture of bioactive chemicals into the host including histamine binders and cytokine inhibitors to mediate host response, complement inhibitors to suppress the host immune response, and anticoagulants to facilitate the blood meal..$^{30,31}$ This results in a painless "bite" and usually prevents an inflammatory response. Injection of saliva occurs over a major part of the attachment time with the blood meal occurring late in the process..$^{32}$ Approximately $75 \%$ of the water taken up during feeding is returned to the host. ${ }^{33}$ When a tick feeds on an animal infected with Borrelia, spirochetes can be ingested, and travel with the hemolymph to the midgut where they remain through subsequent life stages. Ribeiro et al found that $30 \%-55 \%$ of I. scapularis feeding on infected rabbits had detectable spirochetes in their midgut. ${ }^{34}$

When an infected tick takes the next stage blood meal, there is a possibility that pathogenic organisms can be transferred to the new host. Based upon rabbit model experiments, a salivary route for the transfer of Borrelia spirochetes from the midgut to new hosts was proposed in 1987 by Ribeiro et al. The potential for pathogen transmission by regurgitation was considered; however visual and microscopic observation of post feeding saliva found no evidence of this. ${ }^{34}$

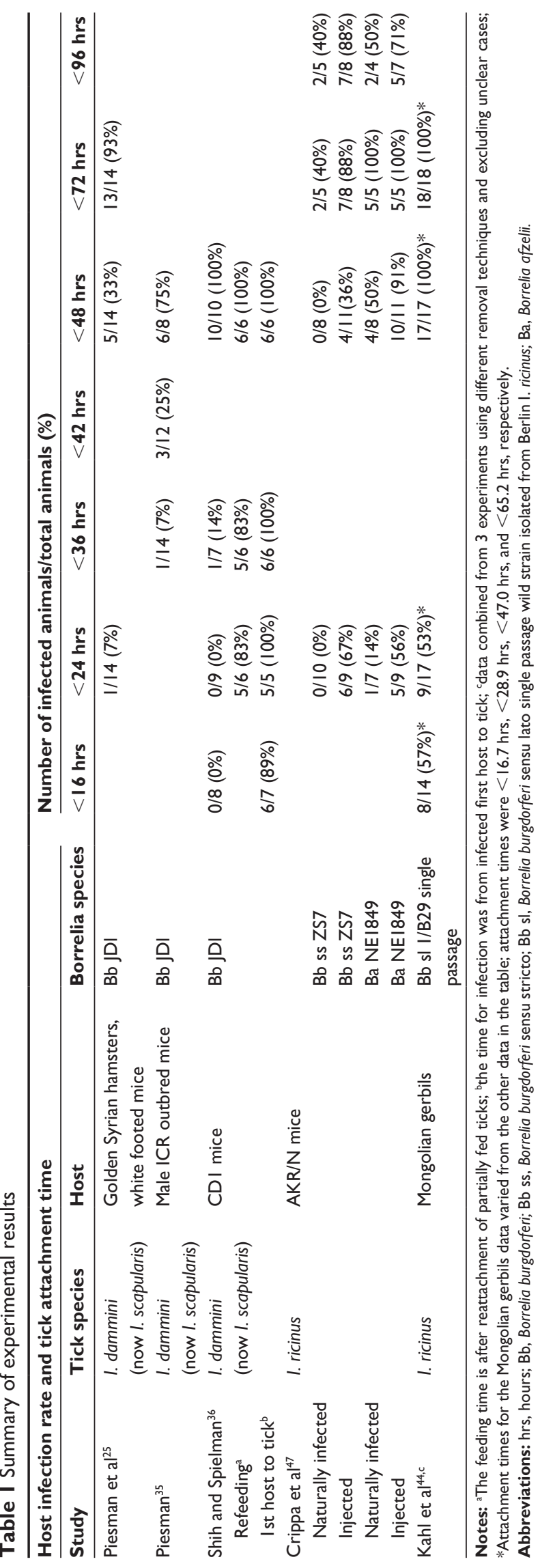




\section{Attachment time experiments}

In a 1987 paper, Piesman et al published data from an animal model study of the time for B. burgdorferi sensu stricto spirochetes to transfer to rodents. They observed an increased risk of infection with longer tick attachment times. However, one of the 14 (7\%) test rodents was infected in $<24$ hours, five of the $14(33 \%)$ in $<48$ hours, and 13 of the 14 (93\%) in $<72$ hours. ${ }^{25}$ These results have a margin of error dependent on the sample size. Using a 95\% confidence level, the confidence interval for transmission within 24 hours is $\pm 13.37 \%$ based on one infection in 14 trials. This indicates that the probability of transmission within 24 hours could be as high as $20.37 \%$. Similarly, the probability of infection within 48 hours has a confidence interval of $\pm 24.63 \%$ with a probability of transmission up to $57.63 \%$. The methodology for determining transmission time was xenodiagnosis, allowing ticks carrying Borrelia spirochetes to feed on test mammals, then attaching naïve ticks to the test animals, and allowing feeding to repletion. The detached ticks were then dissected and ingested spirochetes visualized using microscopy and an indirect fluorescent antibody test.

Results of a second group of experiments reported in 1993 provided similar data, with one of $14(7 \%)$ mice infected in $<36$ hours, three of $12(25 \%)$ infected within 36 hours, and six of eight $(75 \%)$ infected within 48 hours. In these experiments, the method of detection was again by dissection of the xenodiagnostic ticks and visualization using a fluorescent antibody test. ${ }^{35}$

Shih and Spielman, also using animal models reported in 1993 that infection occurred mainly after 2 days of feeding; however, they indicated that in one experiment, $100 \%$ of the mice became infected within 48 hours. It was also found that partially fed ticks would efficiently re-attach to a new host, and in these new hosts transmission occurred in $83 \%$ of cases within 24 hours. ${ }^{36}$ The implication of shorter transmission times in the second host shortly after partial feeding in the first host has relevance to human infection, since partial feeding behavior in ticks has been observed in the natural environment ${ }^{37}$ and could occur within a domestic setting of companion animal ownership.

An explanation was proposed for the reported time delay for infection after tick attachment. It was suggested that the spirochetes need to disseminate from the midgut of the ticks to the salivary glands before being injected by the tick into the epidermis of the host. ${ }^{34,38} \mathrm{~A}$ number of workers have studied the location of spirochetes in ticks including pre-attachment to hosts and found that the bacteria were not always confined to the midgut. Burgdorfer et al in 1983 reported dissemination to other organs in $5.5 \%$ of infected Ixodes ricinus ticks, ${ }^{39}$ and in 1988 reported dissemination in $3.9 \%$ of $I$. scapularis ticks. ${ }^{40}$ In the Ribeiro study that demonstrated a salivary route for transmission, the authors indicated that spirochetes infect other organs as well as the midgut and found 5\% of nonfed female I. ricinus carried the infection in the hemolyth circulating throughout the tick. Histological studies reported by Gern et al in 1990 found that when I. ricinus ticks acquired infection during feeding, the spirochetes multiplied in the midgut, but they noted that some spirochetes penetrated the gut wall of the ticks and caused systemic infection. ${ }^{41} \mathrm{~A}$ study by Piesman et al found infected ticks with bacteria in their salivary glands and other organs at the onset of feeding, and even before feeding began. ${ }^{26}$ Lebet and Gern demonstrated that $11 \%$ of field-collected unfed nymphal ticks had systemic infection, ${ }^{42}$ and Leuba-Garcia et al found that $36 \%$ of unfed adult $I$. ricinus ticks collected in two districts of Switzerland had systemic infection, and they commented that this could impact transmission time in humans. ${ }^{43}$ These results suggest that in cases where the spirochetes are present in the tick salivary glands, they can be injected into the host during the preparatory transfers of antihistamines and anticoagulants prior to the commencement of feeding, ie, immediately after attachment of the tick to the host.

\section{Tick and Borrelia species differences}

A European study by Kahl et al in 1998 indicated that almost $50 \%$ of Mongolian gerbils were infected within 16.7 hours of attachment and $100 \%$ infection rates occurred within 48 hours. ${ }^{44}$ It was suggested that the difference between this work and that reported in the US related to the different species of vector (I. scapularis versus I. ricinus) and pathogen (B. afzelii versus B. burgdorferi sensu stricto). There is now a body of evidence that supports this finding and the impact of both tick and Borrelia species on the attachment time to transmit spirochetes. An extensive study using a total of 1962 I. persulcatus ticks infected with B. garinii and B. afzelii found that Borrelia were frequently found in the salivary glands of unfed ticks and that during the early stage of feeding, the proportion of ticks with spirochetes in the salivary glands did not increase, nor the concentration of spirochetes. The authors concluded that Borrelia migration from the tick gut to the salivary glands during feeding is not necessary for pathogen transmission. ${ }^{45} \mathrm{~A}$ comparative study that included Ixodes dammini (now I. scapularis) from northwest regions of the US and I. persulcatus ticks collected in Russia found the rate of Borrelia infection similar at close to $26 \%$; however, there was a distinct difference between the 
levels of systemic infection including salivary glands with an average five times greater in I. persulcatus (12.9\%) compared to I. scapularis $(2.4 \%){ }^{46}$ The low level of systemic infection found in the North American study with I. scapularis would result in a low probability of having systemically infected field-collected ticks in experiments with small sample sizes, and may account for the observed delay in transmission if few or none of the experimental ticks were systemically infected. Crippa et al when using natural infection in an animal model stated that with B. burgdorferi sensu lato, transmission did not occur in $<48$ hours; however, in experiments using B. afzelii, one of seven (14\%) and four of eight (50\%) mice became infected in $<24$ hours and $<48$ hours, respectively. In experiments using needle inoculation both $B$. burgdorferi and $B$. afzelii infection occurred within 24 hours, with six of nine $(67 \%)$ and five of nine $(56 \%)$ of the mice infected, respectively. ${ }^{47}$ (See Table 1 for a summary of experimental results). A discussion of factors that differentiate animal models from human infection is covered by Hynote et al. ${ }^{48}$ They highlighted the fact that for the animal studies laboratory strains of B. burgdorferi such as B31 were used and that these strain have decreased infectivity compared to the wild strains. Schwan et al in 1988 demonstrated reduced infectivity for all strains tested including B31 after serial passage in culture, ${ }^{49}$ and others have confirmed and expanded on this work. ${ }^{50-52}$ Also, coinfections may result in increased infectivity. ${ }^{53}$

\section{Virulence, infectivity, and outer surface proteins}

There is evidence that spirochetes residing in the salivary glands are far more infective than those residing in the midgut. Lima et al used salivary gland extracts and midgut extracts from I. scapularis, and using needle inoculation determined the probability of infection with different spirochete loads. They found that it required on average 14 times more spirochetes in midgut extracts to infect compared to inoculations with salivary gland extracts. The median infectious dose $\left(\mathrm{ID}_{50}\right)$ was 18 spirochetes with salivary gland extract and 251 with midgut extract. ${ }^{54}$ Zeidner et al demonstrated that there were tick and Borrelia species differences in the dissemination and spirochete load in host organs and that these were probably related to the fact that there were at least five major protein differences between I. scapularis and $I$. ricinus salivary gland lysate. ${ }^{55}$

There are a large number of proteins present in tick saliva and work is needed to understand or clarify the functions of most of them. Two that have received much attention are Outer Surface Protein A (OspA) and Outer Surface
Protein C (OspC). OspA is expressed when the bacteria are within the tick midgut during the dormant phase, and its presence is a requirement for survival in the tick by facilitating adhesion of the spirochete to the midgut wall. ${ }^{56}$ When the tick prepares for the blood meal, OspA is downregulated in preparation for the transit from midgut to the salivary glands and from there to the host. At the same time, OspC is upregulated. ${ }^{57,58}$ The role of OspC is not clear; various functions have been ascribed to the protein, and it may have multifactorial activity including host invasion and dissemination, and for initiation of host infection. ${ }^{59}$ OspC expression and infectivity continues to increase for some days once the spirochetes have invaded host tissues. Though being antigenic, it is eventually downregulated to minimize host antibody response. A major factor in the OspA/OspC complementary expression is ambient temperature, with OspC not present at low temperatures but present at $34^{\circ} \mathrm{C}-37^{\circ} \mathrm{C} .{ }^{57}$ This can be important in the context of transmission time of Borrelia from tick to host. Tick blood feeding behavior includes engagement, the adherence to the host; exploration, the search for a suitable site for attachment; and penetration, where the tick inserts the mouthparts in preparation for feeding. ${ }^{60}$ During the process of exploration when the tick is searching for a suitable site, temperature increase will activate OspA/OspC regulation and begin the process of increased motility and infectivity. Exploration time will be highly variable and depend on how quickly the tick migrates to an optimal site. This time could vary with host animal size, the presence of competing ticks, or rejection of a site as unsuitable. Unattached and un-engorged ticks are routinely observed by deer hunters and pet owners. Hence, exploration time must be included with attachment time to more accurately reflect "time for transmission".

\section{Estimating attachment times in humans}

In the case of animal experiments, it is possible to clearly define the moment of tick attachment, but it is seldom possible in the case of human tick bites. In one study, only $50 \%$ of subjects were able to estimate tick attachment times and then not with an accuracy thought to be better than 24 hours. ${ }^{61}$ Usually the first knowledge of tick attachment is when it is noticed by the victim. The time of presentation to a clinician will be known accurately; however, the time interval between attachment and presentation to a clinician is in general unknown. The consequence is that in the majority of cases, the length of time of attachment to human hosts cannot be determined with accuracy. 
To overcome this problem, there have been proposals for the use of indirect measurement methods. As a tick takes a blood meal, the body becomes engorged and the change in body length has been suggested as a method to estimate the time of attachment. Falco et al studied this in 1996 and used the scutal index, which is the ratio of the length of the hard body plate to the tick body length. ${ }^{24}$ Their published graphical data indicate that the method cannot discriminate between attachment times $<24$ hours, it is poor up to 36 hours, and there is large variability thereafter. This is explained by the blood meal and engorgement taking place during the later stage of attachment. Meiners et $a^{62}$ found that the scutal index estimate for attachment time had poor correlation with the attachment time estimated by the patients. No definitive correlation data were presented for longer attachment times. A similar methodology was studied with I. ricinus where the ratio of the scutum to the distance between the coxae of the fourth pair of legs (coxal index) was used. The method was considered more accurate than the scutal index for attachment times up to 12 hours, and the scutal index more accurate with longer attachment times, though the method predicted times from near 11 hours up to 30 hours for attachment times of 24 hours with nymphal ticks, and with similar results for adults. This poor correlation indicates that neither the scutal index nor the coxal index is useful as a risk indicator.

In cases of human interactions in tick endemic areas, the time of exposure can be narrowed down to a window of opportunity and there are many anecdotal descriptions of tick attachment and transmission of disease in $<24$ hours. Some cases where there is strong evidence of early transmission have been published. ${ }^{48,63}$ A European study documented six cases of culture-confirmed infection where tick attachment was $<6$ hours and nine cases where transmission occurred in $<24$ hours. ${ }^{64}$ Angelov reported a case based on clinical symptoms and positive serology, where infection was transmitted in $<24$ hours and a second case where a patient's conjunctiva was infected by the intestinal contents of a tick. ${ }^{65}$ The case of fecal transmission indicates that other mechanisms including via mucus membranes need to be considered in addition to the conventional intradermal transmission route. Although attachment time was not accurately determined they do give evidence to support early infection. ${ }^{48}$

\section{Conclusion}

The claims that removal of ticks within 24 hours or 48 hours of attachment will effectively prevent LB are not supported by the published data, and the minimum tick attachment time for transmission of LB in humans has never been established.

The definitive experimental data indicate transmission of LD spirochetes in $<16$ hours and frequently in $<24$ hours. No studies have been carried out to characterize transmission with attachment times of $<16$ hours and some did not report any data for $<36$ hours. The animal studies, however, did not comprehend significant variables such as the presence of spirochetes within the salivary glands at the start of feeding, nor the effect of delayed attachment once a tick adheres to a host. Both of these will reduce the time for infectious spirochetes to invade the host compared to the model that requires a change from dormancy to active migration from the midgut to the salivary glands and then to the host. Also, co-feeding and partial feeding of ticks with displacement from companion animals to humans must be considered a factor for rapid transmission of LD. Though the risk of a single occurrence may be small, this is compounded by the possibility of decades of recreation and lifestyle behavior, especially with companion animals or employment in forestry work and farming.

There is evidence that the risk of LB increases with longer tick attachment time, and the advice to remove a tick quickly is sound. However, there are practical problems identifying attached ticks quickly. The size of ticks at all stages of their life cycle makes them difficult to detect even on exposed skin and nearly impossible in areas where there is limited visibility and within the hairline. The painless bite and histamine suppression ensure that many victims are unaware of the tick bite. At clinical presentation it will not normally be possible to determine the attachment time and as discussed this is not the only contributor to infection. Tick and Borrelia species are also determining factors with evidence of faster transmission for European strains. Additionally ticks carry many other diseases including tick-borne encephalitis, human granular ehrlichiosis, babesiosis, bartonellosis, and others. The attachment times for transmission of many of these are unknown, though there is evidence that some are transmitted very quickly.

Therefore, LB infection can never be excluded after a tick bite irrespective of the estimated duration of attachment time.

\section{Acknowledgments}

I would like to thank PK Puri, Imperial College, London, for his support and encouragement and final review, and Dr Victoria Cairns, Oxford, for her proof reading and suggestions with the early version of the manuscript, and to 
the three reviewers for their encouragement and valuable suggestions.

\section{Disclosure}

The author has no financial competing interests. The author was diagnosed with Lyme disease in 2009.

\section{References}

1. Burgdorfer W, Barbour AG, Hayes SF, Benach JL, Grunwaldt E, Davis JP. Lyme disease - a tick-borne spirochetosis? Science. 1982;216(4552): 1317-1319.

2. Afzelius A. Verhandlung der dermatologishen Gesellschaft zu Stockholm [Proceedings of the Dermatological Society in Stockholm]. Arch Dermo Syph. 1910;101:404. German.

3. Buchwald A. Ein Fall von diffuser idiopathischer Hautatrophie [A case of diffuse idiopathic skin atrophy]. Vjschr Derm. 1883;15:553-556. German.

4. Margos G, Hojgaard A, Lane RS, et al. Multilocus sequence analysis of Borrelia bissettii strains from North America reveals a new Borrelia species, Borrelia kurtenbachii. Ticks Tick Borne Dis. 2010;1(4):151-158.

5. Rizzoli A, Hauffe H, Carpi G, Vourc HG, Neteler M, Rosa R. Lyme borreliosis in Europe. Euro Surveill. 2011;16(27):1-8.

6. Rudenko N, Golovchenko M, Grubhoffer L, Oliver JH. Updates on Borrelia burgdorferi sensu lato complex with respect to public health. Ticks Tick Borne Dis. 2011;2(3):123-128.

7. Fukunaga M, Takahashi Y, Tsuruta Y, et al. Genetic and phenotypic analysis of Borrelia miyamotoi sp. nov., isolated from the ixodid tick Ixodes persulcatus, the vector for Lyme disease in Japan. Int J Syst Bacteriol. 1995;45(4):804-810.

8. Sarksyan DS, Platonov AE, Karan LS, et al. Clinical presentation of "new" tick-borne borreliosis caused by Borrelia miyamotoi. Ter Arkh. 2012;84(11):34-41.

9. Hansford KM, Fonville M, Jahfari S, Sprong H, Medlock JM. Borrelia miyamotoi in host-seeking Ixodes ricinus ticks in England. Epidemiol Infect. 2014. Epub 2014 July 14.

10. Jouda F, Perret J-L, Gern L. Density of questing Ixodes ricinus nymphs and adults infected by Borrelia burgdorferi sensu lato in Switzerland: spatio-temporal pattern at a regional scale. Vector Borne Zoonotic Dis. 2004;4(1):23-32.

11. Lani R, Moghaddam E, Haghani A, Chang L-Y, AbuBakar S, Zandi K. Tick-borne viruses: a review from the perspective of therapeutic approaches. Ticks Tick Borne Dis. 2014;5(5):457-465.

12. Zhang X-C, Yang Z-N, Lu B, Ma X-F, Zhang C-X, Xu H-J. The composition and transmission of microbiome in hard tick, Ixodes persulcatus, during blood meal. Ticks Tick Borne Dis. 2014;5(6):864-870.

13. Krause PJ, Telford SR 3rd, Spielman A, et al. Concurrent Lyme disease and babesiosis evidence for increased severity and duration of illness. JAMA. 1996;275(21):1657-1660.

14. Berghoff W. Chronic Lyme disease and co-infections: differential diagnosis. Open Neurol J. 2012;6:158-178.

15. Eshoo MW, Crowder CD, Carolan HE, et al. Broad-range survey of tick-borne pathogens in southern Germany reveals a high prevalence of Babesia microti and a diversity of other tick-borne pathogens. Vector Borne Zoonotic Dis. 2014;14(8):584-591.

16. Herrmann C, Gern L, Voordouw MJ. Species co-occurrence patterns among Lyme borreliosis pathogens in the tick vector Ixodes ricinus. Appl Environ Microbiol. 2013;79(23):7273-7280.

17. Andersson M, Bartkova S, Lindestad O, Råberg L. Co-infection with "Candidatus Neoehrlichia mikurensis" and Borrelia afzelii in Ixodes ricinus ticks in Southern Sweden. Vector Borne Zoonotic Dis. 2013;13(7):438-442.

18. Sperling J, Middelveen M, Klein D, Sperling F. Evolving perspectives on Lyme borreliosis in Canada. Open Neurol J. 2012;6: 94-103.
19. Ebel GD, Kramer LD. Short report: duration of tick attachment required for transmission of Powassan virus by deer ticks. Am J Trop Med Hyg. 2004;71(3):268-271.

20. Saraiva DG, Soares HS, Soares JF, Labruna MB. Feeding Period Required by Amblyomma aureolatum Ticks for Transmission of Rickettsia rickettsii to Vertebrate Hosts. Emerg Infect Dis. 2014;20(9):1504-1510.

21. Lindgren E, Jaenson TGT. Lyme Borreliosis in Europe: Influences of Climate and Climate Change, Epidemiology, Ecology and Adaptation Measures. Copenhagen: World Health Organization; 2006. EU/04/5046.

22. Müller I, Freitag MH, Poggensee G, et al. Evaluating frequency, diagnostic quality, and cost of Lyme borreliosis testing in Germany: a retrospective model analysis. Clin Dev Immunol. 2012;2012: $1-13$.

23. Centers for Disease Control and Prevention. CDC provides estimate of Americans diagnosed with Lyme disease each year [CDC Media Relations Press Release]. Atlanta: Centers for Disease Control and Prevention; Monday, August 19, 2013; 2013. Available from: http: \ www.cdc.gov/media/releases/2013/p0819-lyme-disease.html. Accessed August 23, 2013.

24. Falco RC, Fish D, Piesman J. Duration of tick bites in a Lyme diseaseendemic area. Am J Epidemiol. 1996;143(2):187-192.

25. Piesman J, Mather TN, Sinsky RJ, Spielman A. Duration of tick attachment and Borrelia burgdorferi transmission. J Clin Microbiol. 1987;25(3):557-558.

26. Piesman J, Schneider BS, Zeidner NS. Use of quantitative PCR to measure density of Borrelia burgdorferi in the midgut and salivary glands of feeding tick vectors. J Clin Microbiol. 2001;39(11):4145-4148.

27. Lees A. The sensory physiology of the sheep tick, Ixodes ricinus L. J Expr Biol. 1948;25:145-207.

28. Richter D, Matuschka F, Spielman A, Mahadevan L. How ticks get under your skin: insertion mechanics of the feeding apparatus of Ixodes ricinus ticks. Proc Biol Sci. 2013;280:20131758.

29. Richter D, Matuschka F, Spielman A, Mahadevan L. How ticks get under your skin: insertion mechanics of the feeding apparatus of Ixodes ricinus ticks. Data supplement [video]. Proc Biol Sci. 2013;280:20131758. Available from: https://www.youtube.com/watch?v=wPbIot9tQ6U. Accessed December 10, 2014.

30. Muller-Doblies U, Wikel S. Human reactions to ticks. In: Goodman JL, Dennis DT, Sonenshine DE, editors. Tick Borne Diseases of Humans. Washington, DC: ASM Press; 2005:102-122.

31. Nuttall PA, Labuda M. Saliva-assisted transmission of tick-borne pathogens. In: Bowman AS, Nuttall PA, editors. Ticks: Biology, Disease and Control. 2008th ed. Cambridge: Cambridge University Press; 2008:205-219.

32. Kemp DH, Stone BF, Binnington KC. Tick attachment and feeding: role of the mouthparts, feeding apparatus and salivary gland secretions and the host response. In: Obenchain FD, Galun R, editors. Physiology of Ticks. Oxford: Pergamon Press; 1982:119-168.

33. Campbell E, Burdin M, Hoppler S, Bowman A. Role of an aquaporin in the sheep tick Ixodes ricinus: assessment as a potential control target. Int J Parasitol. 2010;40(1):15-23.

34. Ribeiro JMC, Mather TN, Piesman J, Spielman A. Dissemination and salivary delivery of Lyme disease spirochetes in vector ticks (Acari: Ixodidae). J Med Entomol. 1987;2:201-205.

35. Piesman J. Dynamics of Borrelia burgdorferi transmission by nymphal Ixodes dammini ticks. J Infect Dis. 1993;167(5):1082-1085.

36. Shih CM, Spielman A. Accelerated transmission of Lyme disease spirochetes by partially fed vector ticks. J Clin Microbiol. 1993;31(11): 2878-2881.

37. Balashov Y. Blood sucking ticks (Ixodoidea) - vectors of diseases of man and animals. Misc Publ Entomol Soc Am. 1972;8:161-376.

38. Zung J, Ewengrub S, Rudzinska M, Sepielman A, Telford S, Peisman J. Fine structural evidence for the penetration of the Lyme disease spirochetes Borrelia burgdorferi through the gut and salivary tissue of I. Dammini. Can J Zool. 1989;67:1737-1748. 
39. Burgdorfer W, Barbour AG, Hayes SF, Prter O, Aeschlimann A. Erythema chronicum migrans: a tick-borne spirochetosis. Acta Trop. 1983;40:79-83.

40. Burgdorfer W, Hayes SF, Benach JL. Development of Borrelia burgdorferi in ixodid tick vectors. Ann N Y Acad Sci. 1988;539: $172-179$.

41. Gern L, Zhu Z, Aeschlimann A. Development of Borrelia burgdorferi in Ixodes ricinus females during blood feeding. Ann Parasitol Hum Comp. 1990;65(2):89-93.

42. Lebet N, Gern L. Histological examination of Borrelia burgdorferi infections in unfed Ixodes ricinus nymphs. Exp Appl Acarol. 1994;18(3): 177-183.

43. Leuba-Garcia S, Kramer MD, Wallich R, Gern L. Characterization of Borrelia burgdorferi isolated from different organs of Ixodes ricinus ticks collected in nature. Zentralblatt für Bakteriol. 1994;280:468-475.

44. Kahl O, Janetzki-Mittmann C, Gray J, Jonas R, Stein J, De Boer R. Risk of infection with Borrelia burgdorferi sensu lato for a host in relation to the duration of nymphal Ixodes ricinus feeding and the method of tick removal. Zentralbl Bakteriol. 1998;287:41-52.

45. Moskvitina GG, Korenberg EI, Gorban LI. The presence of Borrelia in the intestines and salivary glands of spontaneously infected adult Ixodes persulcatus Schulze ticks during bloodsucking. Meditsinskaia Parazitol. 1995;3:16-20.

46. Moskvitina GG, Korenberg EI, Spielman A, Shchegoleva TV. The frequency of generalized infection in adult fasting ticks of the genus Ixodes in foci of borreliosis in Russia and the USA. Parazitologiia. 1995;29(5):353-360.

47. Crippa M, Rais O, Gern L. Investigations on the mode and dynamics of transmission and infectivity of Borrelia burgdorferi sensu stricto and Borrelia afzelii in Ixodes ricinus ticks. Vector Borne Zoonotic Dis. 2002;2(1):3-9.

48. Hynote ED, Mervine PC, Stricker RB. Clinical evidence for rapid transmission of Lyme disease following a tickbite. Diagn Microbiol Infect Dis. 2012;72(2):188-192.

49. Schwan TOMG, Burgdorfer W, Garon CF. Changes in infectivity and plasmid profile of the Lyme disease spirochete, Borrelia burgdorferi, as a result of in vitro cultivation. Infect Immun. 1988;56(8):1831-1836.

50. Xu Y, Kodner C, Coleman L, Johnson RC. Correlation of plasmids with infectivity of Borrelia burgdorferi sensu stricto type strain B31. Infect Immun. 1996;64(9):3870-3876.

51. Labandeira-Rey M, Skare JT. Decreased Infectivity in Borrelia burgdorferi Strain B31 is associated with loss of linear plasmid 25 or 28-1. Infect Immun. 2001;69(1):446-455.
52. Purser JE, Norris SJ. Correlation between plasmid content and infectivity in Borrelia burgdorferi. Proc Natl Acad Sci U S A. 2000;97(25): 13865-13870.

53. Mather TN, Telford SR, Moore SI, Spielman A. Borrelia burgdorferi and Babesia microti: efficiency of transmission from reservoirs to vector ticks (Ixodes dammini). Exp Parasitol. 1990;70(1):55-61.

54. Lima CM, Zeidner NS, Beard CB, et al. Differential infectivity of the Lyme disease spirochete Borrelia burgdorferi derived from Ixodes scapularis salivary glands and midgut. J Med Entomol. 2005;42(3): 506-510.

55. Zeidner NS, Schneider BS, Nuncio MS, Gern L, Piesman J. Coinoculation of Borrelia spp. with tick salivary gland lysate enhances spirochete load in mice and is tick species-specific. J Parasitol. 2002;88(6):1276-1278.

56. Pal U, de Silva AM, Montgomery RR, et al. Attachment of Borrelia burgdorferi within Ixodes scapularis mediated by outer surface protein A. J Clin Invest. 2000;106(4):561-569.

57. Schwan TG, Piesman J, Golde WT, Dolan MC, Rosa PA. Induction of an outer surface protein on Borrelia burgdorferi during tick feeding. Proc Natl Acad Sci U S A. 1995;92(7):2909-2913.

58. Schwan TG, Piesman J. Vector interactions and molecular adaptations of Lyme disease and relapsing fever spirochetes associated with transmission by ticks. Emerg Infect Dis. 2002;8(2):115-121.

59. Tilly K, Bestor A, Rosa PA. Lipoprotein succession in Borrelia burgdorferi: similar but distinct roles for OspC and VlsE at different stages of mammalian infection. Mol Microbiol. 2013;89(2):216-227.

60. Waladde S, Rice MJ. The sensory basis of tick feeding behaviour. In: Obenchain F, Galun R, editors. Physiology of Ticks. Oxford: Pergamon Press; 1982:71-118.

61. Sood SK, Salzman MB, Johnson BJ, et al. Duration of tick attachment as a predictor of the risk of Lyme disease in an area in which Lyme disease is endemic. J Infect Dis. 1997;175(4):996-999.

62. Meiners T, Hammer B, Göbel UB, Kahl O. Determining the tick scutal index allows assessment of tick feeding duration and estimation of infection risk with Borrelia burgdorferi sensu lato in a person bitten by an Ixodes ricinus nymph. Int J Med Microbiol. 2006;296(S1):103-107.

63. Patmas M, Remorca C. Disseminated Lyme disease after short-duration tick bite. J Spirochetal Tickborne Dis. 1994;1:77-78.

64. Strle F, Nelson JA, Ruzic-Sabljic E, et al. European Lyme Borreliosis: 231 culture-confirmed cases involving patients with erythema migrans. Clin Infect Dis. 1996;23(1):61-65.

65. Angelov L. Unusual features in the epidemiology of Lyme borreliosis. Eur J Epidemiol. 1996;12(1):9-11.
International Journal of General Medicine

\section{Publish your work in this journal}

The International Journal of General Medicine is an international, peer-reviewed open-access journal that focuses on general and internal medicine, pathogenesis, epidemiology, diagnosis, monitoring and treatment protocols. The journal is characterized by the rapid reporting of reviews, original research and clinical studies across all disease areas.

\section{Dovepress}

A key focus is the elucidation of disease processes and management protocols resulting in improved outcomes for the patient.The manuscript management system is completely online and includes a very quick and fair peer-review system. Visit http://www.dovepress.com/ testimonials.php to read real quotes from published authors. 